Document downloaded from:

http://hdl.handle.net/10251/65190

This paper must be cited as:

Ferrer Sapena, A.; Sánchez Pérez, EA.; González, LM.; Peset Mancebo, MF.; Aleixandre Benavent, R. (2015). Mathematical properties of weighted impact factors based on measures of prestige of the citing journals. Scientometrics. 105(3):2089-2108. doi:10.1007/s11192-015-1741-0.

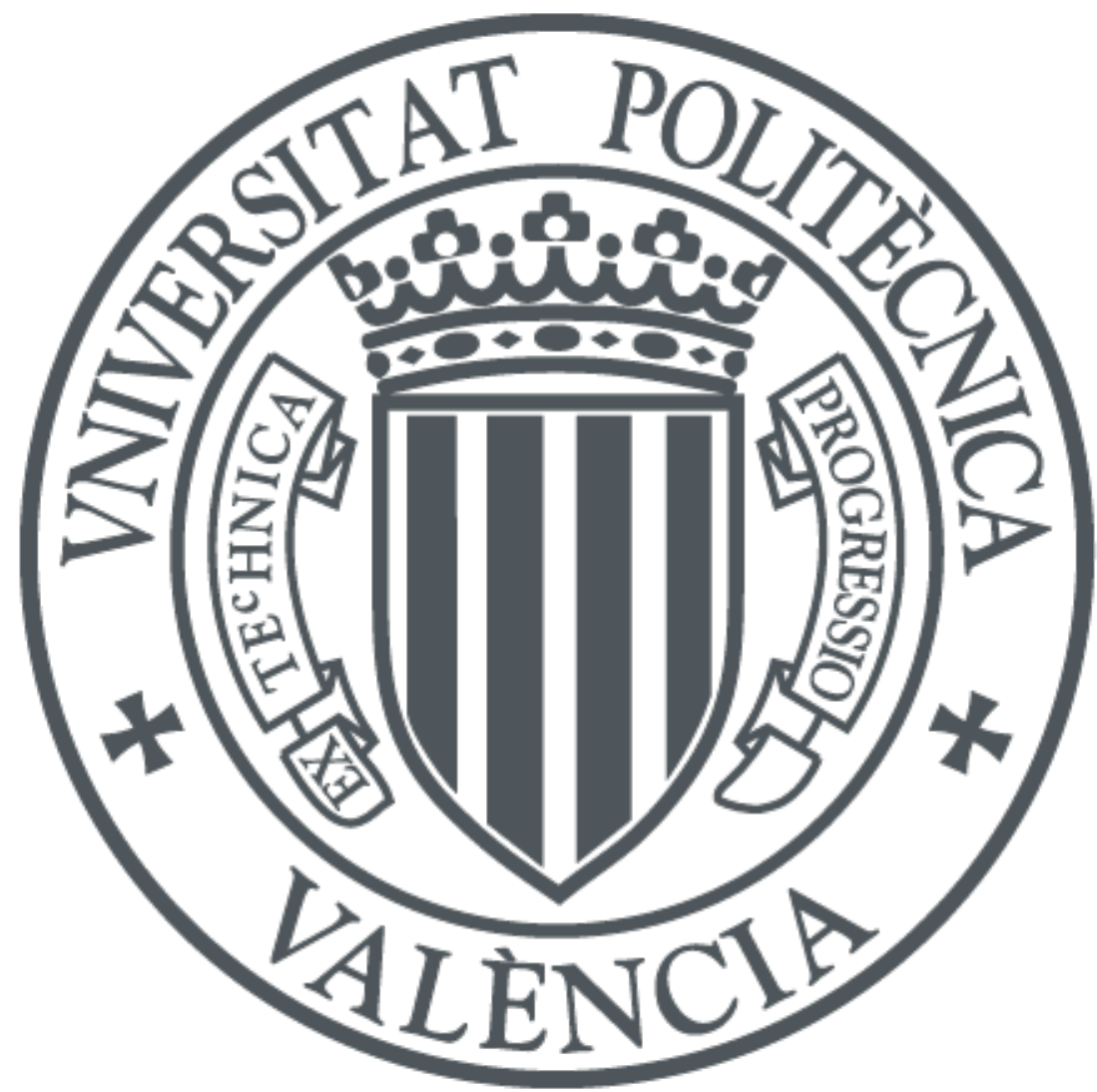

The final publication is available at

http://link.springer.com/article/10.1007/s11192-015-1741-0

Copyright Springer; Akadémiai Kiadó

Additional Information

The final publication is available at Springer via http://dx.doi.org/10.1007/s11192-015-1741-0 


\title{
Mathematical properties of weighted impact factors based on measures of prestige of the citing journals
}

\author{
A. Ferrer-Sapena • E.A. Sánchez-Pérez • \\ L.M. González • F. Peset • \\ R. Aleixandre-Benavent
}

Received: date / Accepted: date

\begin{abstract}
An abstract construction for general weighted impact factors is introduced. We show that the classical weighted impact factors are particular cases of our model, but it can also be used for defining new impact measuring tools for other sources of information - as repositories of datasets -, providing the mathematical support for a new family of altmetrics. We analyze the stability of the ordering induced in a list of journals by the 2-year impact factor $\left(I F_{2}\right)$ when the criterium for the ordering is changed to be defined by the numerical value of a new weighted impact factor, in which $I F_{2}$ is used for defining the weights. We prove that, if we assume that the weight associated to a citing journal increases with its $I F_{2}$, then the ordering given in the list by the new weighted impact factor coincides with the order defined by the $I F_{2}$. We give a quantitative bound for the errors committed. We also show and analyze two examples of weighted impact factors defined by weights associated to the prestige of the citing journal for the fields of MATHEMATICS and MEDICINE, GENERAL and INTERNAL.
\end{abstract}

Keywords Impact factor · weighted · stability · ordering · mathematics · altmetrics

PACS C20 - C65 - C80

Mathematics Subject Classification (2000) 94A15 $\cdot 94 \mathrm{~A} 17 \cdot 28 \mathrm{E} 99$

\footnotetext{
- A. Ferrer-Sapena. Corresponding author. Instituto de Diseño y Fabricación, Universitat Politècnica de València. Camino de Vera s/n, 46022 Valencia, Spain. Tel.: +34-963879392, Fax: +34-963879390. E-mail: anfersa@upv.es

- E.A. Sánchez-Pérez. Instituto Universitario de Matemática Pura y Aplicada, Universitat Politècnica de València. Camino de Vera s/n, 46022 Valencia, Spain. E-mail: easancpe@mat.upv.es

· L.M. González. F.C.A.F.E. Universidad de Valencia. Gascó Oliag, 3, 46010 Valencia, Spain. E-mail: luis.m.gonzalez@uv.es

· F. Peset. Instituto de Diseño y Fabricación, Universitat Politècnica de València. Camino de Vera s/n, 46022, Valencia, Spain. E-mail: mpesetm@upv.es

- R. Aleixandre-Benavent. C.S.I.C. Camino de Vera s/n, 46022 Valencia, Spain. E-mail: Rafael.Aleixandre@uv.es
} 


\section{Introduction}

Measuring citations of published papers is an important tool for analyzing the dynamics of the scientific activity. In particular, the Thomson-Reuters 2-year impact factor is nowadays considered as a useful instrument that reflects (up to a point) the scientific prestige of a journal (Altmann and Gorman 1998; Garfield 2006; Pinto and Andrade 1999; Saha et al. 2003). In the computation of such index, each citation from a journal belonging to a given list has the same value. In order to adapt this parameter to become a better tool for measuring the "prestige" of a journal - in the sense of the scientific impact that can be measured by citations - , a natural modification to make is to give a different weight to each citation. These weights reflect the "quality" of this citation (Buela-Casal 2003; Habibzadeh and Yadollahie 2008; Waltman and van Eck 2008; Zitt and Small 2008; Zyczkowski 2010). This is on the basis of the definition of the so called weighted impact factors. Probably the first formal development of this idea that can be found in the scientific literature was given in the relevant paper by Pinski and Narin (1976). Actually, a lot of impact-factor-like tools may be considered as particular cases or extensions of this idea. The reader can find a lot of classical and new papers on this subject (see for example Leydesdorff and Opthof 2010; Moed 2010; TorresSalinas and Jimenez-Contreras, 2010; Waltman et al. 2013; Zitt 2011; Zitt and Small 2008). More general weights for defining impact measuring tools could be defined using recent analytical tools, taking into account the specific scientific field (Aleixandre Benavent et al. 2007; Dorta-Gonzalez 2013; DortaGonzalez et al. 2014; Egghe and Rousseau 2002; Li et al. 2013; Owlia et al. 2011; Ruiz Castillo and Waltman 2015).

The emergence of new information sources and the need of measuring their impact make necessary to consider a more abstract version of these weighted impact factors. This is the aim of the present paper. For example, we will show that our mathematical formulation can be easily adapted to allow the definition of impact factors for open databasis that are used to compute new information also available in other open databasis. In this paper we propose a general mathematical model for measuring the impact of scientific information, and we study some of its properties.

As an application, in the second part of the paper we analyze the standard weighted impact factors with weights coming from the values of $I F_{2}$ of the citing journals. For the computation of $I F_{2}$, it is implicitly assumed that all the citing journals have the same prestige, and so all the citations have the same weight. However, using $I F_{2}$ for giving weights that define a new impact factor would change the ordering of the list of journals previously ordered by $I F_{2}$. In this paper, we analyze in two concrete cases (two Thomson-Reuters lists of journals of two different scientific areas) if there is a "hidden citing rule", in the sense that journals with high impact factor cite often journals of high impact factor, while the ones with low impact factor cite often journals with low impact factor. We will prove some mathematical consequences on the 
weighted impact factors that hold if this increasing property is satisfied for a given impact factor, in particular for $I F_{2}$.

We will show in this paper that if this particular "increasing" citing behavior of journals is followed by a majority of journals in a list, then changing the $I F_{2}$ by some weighted impact factor does not add any benefit, since the final ordering induced in the list of journals is the same as the one induced by $I F_{2}$ (Proposition 1). We will provide two particular examples of this behavior for the Thomson-Reuters list of journals of MATHEMATICS and MEDICINE, GENERAL AND INTERNAL.

Summing up, our main objective is to analyze the mathematical properties of weighted impact factors when the weight of each citation is given by an index that measures the prestige of the journal where the citing paper is published. We also show that our model - and then its formal consequences - can also be used for measuring the impact of repositories of datasets on other repositories. We will center our attention in the changes in the ordering that the resulting weighted impact factor produces in a given list of journals previously ordered by the usual 2-year impact factor, specially when the list satisfies the increasing property.

We also provide some error bounds and some elaborated examples.

\section{The mathematical model for weighted impact factors}

Some modifications of the usual Impact Factor now in use have been proposed in recent times in order to take into account the behavior of the citing journals besides the properties of the cited ones (Buela-Casal 2003; Habibzadeh and Yadollahie 2008; Waltman and van Eck 2008). The usual way of doing it is by adding some multiplicative weights in the definition of the new indexes that attempt to modulate the effect of the particular characteristics of the citing journals (see for example Zitt and Small 2008, and the references therein). In this section we present step by step a model that allows to understand this kind of weighted impact factor in a more abstract way than usual, in order to obtain some general properties regarding its mathematical behavior. Throughout the paper, and for the aim of clarity, we will write $I F_{2}$ for the Thomson-Reuters 2-year Impact Factor, and $I S$ for a generic weighted impact factor. Let us show how to define such an impact factor $I S$.

(1) Consider a set of journals $\mathcal{R}$ containing a set $\mathcal{A}$ of cited articles and a set $\mathcal{C}$ of citing articles. We want to construct a mathematical representation of the way the set $\mathcal{C}$ cites the set $\mathcal{A}$. In the abstract formulation that we will construct, $\mathcal{A}$ is in general a set of information items that is used for constructing a new set of information items $\mathcal{C}$. Both sets are supposed to have associated $\sigma$-algebras of subsets $\Sigma_{\mathcal{A}}$ and $\Sigma_{\mathcal{C}}$, that is $\left(\mathcal{A}, \Sigma_{\mathcal{A}}\right)$ and $\left(\mathcal{C}, \Sigma_{\mathcal{C}}\right)$ are measurable spaces. If $A$ is a finite set, we write as usual $|A|$ for the number of elements of $A$.

(2) Let us write $\mathbf{F}(\mathcal{C}, \mathbb{R})$ for the space of all functions from $\mathcal{C}$ to $\mathbb{R}$, the field of the real numbers. We consider a function $\phi: \mathcal{A} \rightarrow \mathbf{F}(\mathcal{C}, \mathbb{R}), \mathcal{A} \ni a \mapsto$ 
$\phi_{a}(c) \in \mathbb{R}, c \in \mathcal{C}$, representing how each article $a \in \mathcal{A}$ is cited by the element $c$ of the set $\mathcal{C}$, or in a more general sense, how the elements of $\mathcal{C}$ influence the citation of a given paper $a \in \mathcal{A}$ (weight function). In the normal cases, the function $\phi$ can be given by the weight we want to associate in our model to the citation by an article $c \in \mathcal{C}$ to an article $a \in \mathcal{A}$. In the abstract case, this function represents the weight that we want to give in our index to the use of a given information item $a \in \mathcal{A}$ by a given information item $c \in \mathcal{C}$.

(3) A function $E: \phi(\mathcal{A}) \rightarrow \mathbb{R}$ evaluating each real function $\phi_{a}$ for a given $a \in \mathcal{A}$, which represents the contribution of all the citing elements of $\mathcal{C}$ to the value of the impact that we want to give to the paper $a$. The standard case is given just by summation, i.e.

$$
E\left(\phi_{a}\right):=\sum_{c \in \mathcal{C}} \phi_{a}(c)
$$

that is, the weighted sum of the times that $a$ is cited by the elements of $\mathcal{C}$ : the weights are given by $\phi_{a}(c)$. More generally, it gives the measure that we want to give to the use of an information item $a \in \mathcal{A}$ in the obtention of the elements of $\mathcal{C}$, that is, how relevant is the contribution of $a$ for obtaining all the data of $\mathcal{C}$. The formula representing this is

$$
E\left(\phi_{a}\right)=\int_{\mathcal{C}} \phi_{a}(c) d \mu(c)
$$

for a given fixed measure $\mu$ on $\Sigma_{\mathcal{C}}$.

(4) For obtaining our new impact factor for a cited journal $r$, it is enough to compute the mean of all the values of $E\left(\phi_{a}\right)$ for all the articles $a \in r$,

$$
I S(r)=\frac{\sum_{a \in r} E\left(\phi_{a}\right)}{|r|}=\frac{\sum_{a \in r} \sum_{c \in \mathcal{C}} \phi_{a}(c)}{|r|},
$$

where $|r|$ is the number of articles in the journal $r$. The formula for general sets $\mathcal{A}$ and $\mathcal{C}$ of information items is in this case

$$
I S(A)=\int_{A} E\left(\phi_{a}\right) d \tau_{A}(a)=\int_{A}\left(\int_{\mathcal{C}} \phi_{a}(c) d \mu(c)\right) d \tau_{A}(a),
$$

where $A \subseteq \mathcal{A}$ and $\tau_{A}$ is a probability measure on the restriction of $\Sigma_{\mathcal{A}}$ to A.

Note that formula (1) coincides with this general expression for $I S$ when $A$ is the set of all the papers $a$ in a journal $r$ and $\tau_{A}$ is the probability measure given by $\tau_{A}(\{a\})=1 /|A|$ for all $a \in A$, and $\tau_{A}(\{a\})=0$ for $a \notin A$. The measure $\mu$ is just the counting measure over $\mathcal{C}$, i.e. the measure that satisfies that $\mu(\{c\})=1$ for each $c \in \mathcal{C}$.

As a consequence of Fubini's Theorem - or simply using the fact that all the integrals appearing above are finite sums in the discrete case-, we get the following equivalent expression for $I S$.

$$
I S(A)=\int_{\mathcal{C}}\left(\int_{\mathcal{A}} \phi_{a}(c) d \tau_{A}(a)\right) d \mu(c), \quad A \in \Sigma_{\mathcal{A}} .
$$


The simplest - and in a sense standard - example, is given by the way citations are considered in the definition of the $I F_{2}$ for a set of journals $\mathcal{R}$ in a given year $n$. In this case, the set $\mathcal{A}$ is given by the papers published by the journals in $\mathcal{R}$ in the years $n-2$ and $n-1$, and $\mathcal{C}$ is the set of the articles published in the year $n$ in a fixed set of journals $\mathcal{R}$, for example, the ones appearing in the Thomson-Reuters Web of Science Core Collection. Let us write in this case $e$ for the function $\phi$ in the step (3) above, that is defined by

$$
e_{a}(c)=1 \quad \text { if } a \in \mathcal{A} \text { is cited by } c \in \mathcal{C} \text {, and } e_{a}(c)=0 \text { otherwise. }
$$

The number of citations of each article $a \in \mathcal{A}$ by the elements of $\mathcal{C}$ is given by

$$
E\left(e_{a}\right)=\sum_{c \in \mathcal{C}} e_{a}(c)=\sum_{c \in \mathcal{C}_{a}} 1=\left|C_{a}\right|
$$

where $C_{a}$ is the subset of papers in $\mathcal{C}$ citing the article $a$ and $\left|C_{a}\right|$ is the number of elements of $C_{a}$. The standard Thomson-Reuters (2-year ) impact factor for a journal $r \in \mathcal{R}$ is then given by

$$
I F_{2}(r)=\frac{\sum_{a \in r} E\left(e_{a}\right)}{|r|}=\frac{\sum_{a \in r}\left|C_{a}\right|}{|r|} .
$$

In order to justify the abstract definition of the model given in this section for measuring the impact of information sources based on the Lebesgue integral with respect to positive measures, let us provide two examples of weighted impact measures for data repositories. The integrals cannot be changed by finite sums in them. Our aim is to show that our model can be used as a formal support for some aspects of the nowadays relevant topic of the measuring of the impact of items of scientific information others than the usual journal papers: the altmetrics. Actually, some tools for measuring the impact of datasets of diverse sources are being testing at that time (see NISO 2014). For instance, one of these purposes is to measure the impact of datasets by means for the actual bites that are downloaded from them and the times that the associated metadata are checked by the usuaries. Our mathematical formulation fits adequately with this aim.

\subsection{Example: a storm risk index}

Consider a data repository $\mathcal{A}$ defined by a finite class $A_{1}, \ldots, A_{n}$ of data sets. Suppose that we are interested in evaluating the impact of some particular subsets of the total set of information contained in all of them, that is, subsets of $\cup_{i=1}^{n} A_{i}$. We want to measure the impact of such a subset $A$ with respect to a different data repository $\mathcal{C}$, that contains the data sets $C_{1}, \ldots, C_{m}$. It is supposed that the data contained in $\cup_{j=1}^{m} C_{j}$ are obtained by using the data of $\cup_{i=1}^{n} A_{i}$ as primary information, and are, in a sense "elaborated" or "second order" data. Let us give a concrete example of this situation. Assume that $\mathcal{A}$ contains all the average values of the temperature of the water of the sea at each 
point of a certain region in a year, indexed by the geographical coordinates of the point. Assume that the elements of $\mathcal{C}$ are the data of the weather forecast for the region at every second $t$ in a year, including a predictive index $\psi$ between 0 and 1 that indicates the risk of storm at $t$. The data corresponding to the index $\psi$ are then naturally indexed by the value of $t$.

Suppose we want to evaluate the impact that a particular area $A$ of the region - defined by the coordinates of its points - has for the evaluation of the storm risk at some period of time, that is represented by a particular subset of $\mathcal{C}$. Depending on the weather at a given $t$, the algorithm for computing $\psi$ uses the information coming from different points of the region covered by $\mathcal{A}$. This selection of the points is given at each $t$ by functions $g_{t}: \mathcal{A} \rightarrow[0,1]$ that gives the value 1 to the points that are more important for the storm risk index at the time $t$-depending for example on the direction of the wind-, and 0 to the non relevant points of the region for the computation of $\psi$. Thus, we have that $\psi(t)=\psi\left(t, g_{t}\right)$. This allows to define the impact factor $S R$ of $A$-that plays the role of the "cited journal" — with respect to the subset that provides the storm risk data in the time interval $\left[t_{0}, t_{1}\right] \subset \mathcal{C}$ - that plays the role of the set of "citing journals" — following the formula (2), as

$$
S R(A)=\int_{\mathcal{A}}\left(\int_{t_{0}}^{t_{1}} g_{t}(a) d t\right) \frac{d \mu_{A}(a)}{\mu(A)},
$$

where $d t$ is the usual Lebesgue measure on the real line and $\mu_{A}$ is the Lebesgue measure given by the restriction to the subset $A$ of Lebesgue measure on $\mathbb{R}^{2}$. Clearly, none of these integrals can be written as a sum. However, if $A$ and $B$ are two areas of the region that we are studying, $S R(A) \leq S R(B)$ means that the area $A$ is less relevant for the computation of the storm risk index than the area $B$, and a value of $S R(A)$ near to 0 would justify not considering it for the weather forecast regarding storm risk.

\subsection{Example: a time decreasing impact factor}

Let us explain a different example of the general situation we want to develop. Assume that there is a continuous data set $\mathcal{A}$ indexed by the numbers of the positive part of the real line. Suppose that the elements of other dataset $\mathcal{C}$ are indexed by the numbers of the interval $[0,1]$, that represents the time from the last second of $2015(s=0)$ to the first second of the same year $(s=1)$. It is supposed that the interest of the data of $\mathcal{A}$ decreases exponentially for the computation of the data of $\mathcal{C}$ as time goes, starting from the last second of $2014(t=0)$. The subsets of the dataset $\mathcal{A}$ play the role of the cited journals, and the ones of $\mathcal{C}$ play the role of the citing journals. The "prestige" of the citations of the elements of $\mathcal{C}$ to the subsets of $\mathcal{A}$ decreases also when the times goes, following in this case the function $2(1-c)$. A convenient impact factor for any bounded measurable! subset $A \subseteq \mathcal{A}$ may be then defined as

$$
E X(A)=\int_{0}^{1}\left(\int_{0}^{+\infty} e^{-a} 2(c-1) \frac{d \mu_{A}(a)}{\mu(A)}\right) d \mu(c),
$$


where $\mu$ is Lebesgue measure and $\mu_{A}$ is also this measure when restricted to $A$. Again, this index cannot be written as a discrete sum; by the construction, it seems more natural to use the second integral formula (3) in this case.

\section{Some stability properties of weighted impact factors}

\subsection{Fundamental bounding inequalities for abstract weighted citation indexes}

In this section we establish some properties of the class of weighted impact factors. Let $\mathcal{A}$ and $\mathcal{C}$ a pair of sets of data as in Section 2, that is, the elements of $\mathcal{A}$ are used for obtaining the ones of $\mathcal{C}$. Let $(a, c) \rightarrow \phi_{a}(c) \in \mathbb{R}^{+}$be a weight function defining an impact factor that we call $I S$ as explained in the previous section. Let us define also the standard impact factor $I S T$ by using as weights the functions $c \rightarrow e_{a}(c)$ given in Section 2, that has the value 1 for $a \in \mathcal{A}$ if it is used for obtaining $c \in \mathcal{C}$, and 0 otherwise. That is,

$$
\operatorname{IST}(A):=\int_{\mathcal{C}}\left(\int_{A} e_{a}(c) d \tau_{A}(a)\right) d \mu(c), \quad A \subseteq \mathcal{A} .
$$

Clearly, for every $a \in A$ and $c \in \mathcal{C}, \phi_{a}(c)=\phi_{a}(c) e_{a}(c)$, since every weight $\phi_{a}(c)$ is 0 if $a$ is not used for obtaining $c$ (in the "journals case", if $c$ does not cite a). This gives the index $I F_{2}$ for the usual situation of the Thomson-Reuters journal citation index.

We are interested in computing bounds for the difference among a general $I S$ and $I S T$. We have that for every measurable set $A \subseteq \mathcal{A}$,

$$
\begin{aligned}
|I S(A)-I S T(A)| & =\left|\int_{\mathcal{C}}\left(\int_{A} \phi_{a}(c) d \tau_{A}(a)\right) d \mu(c)-\int_{\mathcal{C}}\left(\int_{A} e_{a}(c) d \tau_{A}(a)\right) d \mu(c)\right| \\
& =\int_{\mathcal{C}}\left(\int_{A}\left(\phi_{a}(c)-1\right) e_{a}(c) d \tau_{A}(a)\right) d \mu(c),
\end{aligned}
$$

and so we obtain

$$
|I S(A)-I S T(A)| \leq \int_{\mathcal{C}}\left(\int_{A}\left|\phi_{a}(c)-1\right| e_{a}(c) d \tau_{A}(a)\right) d \mu(c) .
$$

This provides two different estimates for the difference, depending on how we bound this integral. On the one hand, we have that

$$
(4) \leq \sup _{a \in A, c \in \mathcal{C}}\left|\phi_{a}(c)-1\right| \cdot \int_{\mathcal{C}}\left(\int_{A} e_{a}(c) d \tau_{A}(a)\right) d \mu(c),
$$

that is,

$$
|I S(A)-I S T(A)| \leq \sup _{a \in A, c \in \mathcal{C}}\left|\phi_{a}(c)-1\right| \cdot I S T(A) .
$$

On the other hand, using the Cauchy-Schwartz inequality, we obtain

$$
(4) \leq \int_{\mathcal{C}}\left(\int_{A}\left|\phi_{a}(c)-1\right|^{2} e_{a}(c) d \tau_{A}(a)\right)^{1 / 2}\left(\int_{A} e_{a}(c)^{2} d \tau_{A}(a)\right)^{1 / 2} d \mu(c)
$$




$$
\begin{gathered}
\leq\left(\int_{\mathcal{C}}\left(\int_{A}\left|\phi_{a}(c)-1\right|^{2} e_{a}(c) d \tau_{A}(a)\right) d \mu(c)\right)^{1 / 2}\left(\int_{\mathcal{C}}\left(\int_{A} e_{a}(c) d \tau_{A}(a)\right) d \mu(c)\right)^{1 / 2} \\
=\left(\int_{\mathcal{C}}\left(\int_{A}\left|\phi_{a}(c)-1\right|^{2} e_{a}(c) d \tau_{A}(a)\right) d \mu(c)\right)^{1 / 2} \cdot \operatorname{IST}(A)^{1 / 2} .
\end{gathered}
$$

Therefore,

$$
|I S(A)-I S T(A)| \leq\left(\int_{\mathcal{C}}\left(\int_{A}\left|\phi_{a}(c)-1\right|^{2} e_{a}(c) d \tau_{A}(a)\right) d \mu(c)\right)^{1 / 2} \cdot \operatorname{IST}(A)^{1 / 2} .
$$

These formulas provide the main tools for estimating the differences among the values of a weighted impact factor $I S$ and the standard one $I S T$, and can be used for analyzing the gain in substituting the standard index by a new weighted one. Note that no assumption has been necessary in the definition of $I S$ for these inequalities to hold, so they can be used in any situation which could be represented using our abstract construction. In the next subsection we come back to the case of weighted index for citations among papers and journals.

\subsection{Weighted impact factors for citations among journals}

In what follows, we will center our attention in the case of weighted impact factors for journals of a particular class that is directly related to the impact factor $I F_{2}$ and the weighted versions that can be derived from it. Consider a set of journals $\mathcal{R}$, and suppose that the sets $\mathcal{A}$ and $\mathcal{C}$ are defined by papers published in the journals $r$ of $\mathcal{R}$. Let us introduce the characteristic functions $\chi_{r}: \mathcal{C} \rightarrow \mathbb{R}, r \in \mathcal{R}$, that are given by $\chi_{r}(c)=1$ if $c \in r$ and 0 otherwise.

The class of weights we want to consider is defined by the class of functions $\phi_{a}^{s}(\cdot)$ given by

$$
\phi_{a}^{s}(c)=\sum_{r \in \mathcal{R}} \alpha_{2}(r) e_{a}(c) \chi_{r}(c),
$$

where $\alpha_{2}(r)$ is a weight depending only on the journal $r$, and $e_{a}$ is defined as in Section 2. Our aim is to model the idea of considering citations coming from journals with high Thomson-Reuters 2-year Impact Factor as more relevant - that is, with bigger value of $\alpha_{2}(r)$-, than citations coming from journals with low $I F_{2}$ for the computation of the new impact factor. In other words, the weights will be based on a measure of the prestige (the value of $I F_{2}$ ) of the journal where the citing paper is published. We define them as $\alpha_{2}(r):=$ $I F_{2}(r) / \overline{I F_{2}}$, where $\overline{I F_{2}}$ is the mean of all the values of $I F_{2}(r)$ for all $r \in \mathcal{R}$, the set of journals that define the set $\mathcal{C}$ that we want to consider (see Egghe and Rousseau (2002) for this definition).

Using the formulation given in Section 2, for a given journal $r_{0}$ - which is identified with a set of cited journals in $\mathcal{A}$-, we have

$$
I S_{2}\left(r_{0}\right):=\int_{\mathcal{C}}\left(\int_{\mathcal{A}} \phi_{a}^{s}(c) d \tau_{r_{0}}(a)\right) d \mu(c)
$$




$$
=\int_{\mathcal{C}}\left(\int_{\mathcal{A}}\left(\sum_{r \in \mathcal{R}} \alpha_{2}(r) e_{a}(c) \chi_{r}(c)\right) d \tau_{r_{0}}(a)\right) d \mu(c),
$$

and so

$$
I S_{2}\left(r_{0}\right)=\frac{1}{\left|r_{0}\right|}\left(\sum_{a \in r_{0}}\left(\sum_{r \in \mathcal{R}}\left(\sum_{c \in r} \alpha_{2}(r) e_{a}(c)\right)\right)\right) .
$$

Let us estimate the difference between the usual $I F_{2}$ and $I S_{2}$. On one hand, taking into account that $I S T$ is in this case $I F_{2},(5)$ gives for a given cited journal $r_{0}$

$$
\left|I F_{2}\left(r_{0}\right)-I S_{2}\left(r_{0}\right)\right| \leq \max _{r \in \mathcal{R}}\left|\alpha_{2}(r)-1\right| \cdot I F_{2}\left(r_{0}\right) .
$$

On the other hand, (6) gives the following quadratic estimate for the difference.

$$
\begin{gathered}
\left|I F_{2}\left(r_{0}\right)-I S_{2}\left(r_{0}\right)\right| \\
\leq\left(\frac{\sum_{a \in r_{0}} \sum_{r \in \mathcal{R}}\left(\sum_{c \in r}\left|\alpha_{2}(r)-1\right|^{2} e_{a}(c)\right)}{\left|r_{0}\right|}\right)^{1 / 2} \cdot I F_{2}\left(r_{0}\right)^{1 / 2} .
\end{gathered}
$$

These results can be used for estimating the influence in the ordering of a list of journals by the impact factor when a weighted index $I S_{2}$ is used instead of the standard $I F_{2}$.

\section{Weighted impact factors behaving as $I F_{2}$ for ordering a list of journals}

In this section we analyze the consequence on the ordering of a list of journals based on a weighted impact factor as $I S$ when compared with the corresponding ordering of the list based on $I F_{2}$. Our purpose is to study when a given new index $I S$ defined following our procedure is increasing with respect to the order given by the usual 2-year impact factor $I F_{2}$, i.e. when for a given couple of journals $r_{1}$ and $r_{2}$, we have that

$$
I F_{2}\left(r_{1}\right)<I F_{2}\left(r_{2}\right) \Rightarrow I S\left(r_{1}\right) \leq I S\left(r_{2}\right) .
$$

Clearly, if $I S$ has this property, then the ordering induced by $I F_{2}$ on the set of journals is the same as the one that induces $I S$. This means that this new index is giving no new information on the impact ordering of the set of journals.

We will center our attention in the case when $I S$ is defined as the weighted index $I S$ explained in the previous section, that is given by the weights $\alpha_{2}(r)$ affecting the number of citations represented by the functions $\phi_{a}^{s}$. In order to gain some generality, we will consider weighted indexes as $I S_{\alpha}$ for a general weight function $\alpha$ that represents in some sense a measure of the prestige of the citation.

Notation warning: Note that the generic impact factor IS can be considered also as an index that gives an order to set of journals $\mathcal{R}$-by the decreasing values of IS of the elements in $\mathcal{R}$-, and as a weight $\alpha$, since its numerical 
value is used sometimes to define the weights of a new weighted impact factor. This is the reason we use the terms impact factor, index and weight for the same function, depending on the role it plays in the particular case that it is being used.

Let us say that an impact factor $I S$ has the increasing property if journals with high impact factor tend to receive citations from articles from journals with high impact factor, and reciprocally, journals with low impact factor are cited more often by articles that are published in journals with low impact. Let us formalize this notion.

- Consider a set of journals $\mathcal{R}$ and consider in it an impact factor $I S$. We order $\mathcal{R}$ by the values of $I S$ to produce an ordered list, that is, the first journal is the one with the smallest value of $\alpha$ and the last one the one with the biggest. If there are journals with the same value of $\alpha$ we order them alphabetically.

- Let $n=|\mathcal{R}|$. For each journal $r \in \mathcal{R}$, we associate to it an $n$-fold vector $\beta(r):=\left(\beta_{i}(r)\right)_{i=1}^{n} \in \mathbb{R}^{n}$, where $\beta_{i}$ is defined as

$$
\beta_{i}(r)=N\left(r_{i}, r\right) /|r|,
$$

where $r_{i}$ is the $i$-th journal in the list, $N\left(r_{i}, r\right)$ is the number of citations from articles in $r_{i} \in \mathcal{R}$ to any article in $r$, and $|r|$ the number of articles in

- Let us define the following ordering for the sequences in $\mathbb{R}^{n}$. For two sequences $\left(\lambda_{i}\right)_{i=1}^{n}$ and $\left(\gamma_{i}\right)_{i=1}^{n}$ of non-negative numbers, we write $\left(\lambda_{i}\right)_{i=1}^{n} \triangleleft$ $\left(\gamma_{i}\right)_{i=1}^{n}$ if $\left(\gamma_{i}\right)_{i=1}^{n}$ can be written as a sum of $n$ non-negative sequences $\sum_{j=1}^{n}\left(\gamma_{i}^{j}\right)_{i=1}^{n}=\left(\gamma_{i}\right)_{i=1}^{n}$ such that

$$
\lambda_{i} \leq \sum_{k \geq i} \gamma_{k}^{i}
$$

for every $i \in\{1, \ldots, n\}$. It can be proved that this definition defines an order relation on the set of non-negative sequences of $\mathbb{R}^{n}$.

Definition 1 We say that a list of journals $\mathcal{R}$ ordered by an impact factor $I S$ has the increasing property with respect to $I S$ if for each pair of elements $r, r^{\prime}$ in the list, it is satisfied that, if $I S(r)<I S\left(r^{\prime}\right)$, then $\beta(r) \triangleleft \beta\left(r^{\prime}\right)$ then $I S(r) \leq I S\left(r^{\prime}\right)$, where the sequences $\beta(\cdot)$ are ordered by means of $I S$ as explained above.

Let us show with a simple example that this formal definition corresponds to the concept that we want to model.

Example 1 Suppose that we have a set of three journals $\mathcal{R}=\left\{r_{1}, r_{2}, r_{3}\right\}$, and let an impact factor $I$ for it that is given by $I\left(r_{i}\right)=i, i=1,2,3$. Suppose that

(1) the journal $r_{1}$ publishes 10 papers and receives 2 citations from $r_{1}, 1$ from $r_{2}$ and 0 from $r_{3}$, that is, $N\left(r_{1}, r_{1}\right)=2, N\left(r_{2}, r_{1}\right)=1$ and $N\left(r_{3}, r_{1}\right)=0$. 
(2) The journal $r_{2}$ publishes 20 papers and receives only 8 citations from $r_{2}$, that is $N\left(r_{1}, r_{2}\right)=0, N\left(r_{2}, r_{2}\right)=8$ and $N\left(r_{3}, r_{2}\right)=0$.

(3) The journal $r_{3}$ publishes 30 papers and receives 5 citations from $r_{2}$ and 20 citations from itself, that is $N\left(r_{1}, r_{3}\right)=0, N\left(r_{2}, r_{3}\right)=5$ and $N\left(r_{3}, r_{3}\right)=20$.

Due to the values of $I$, the list is ordered as $r_{1} \leq r_{2} \leq r_{3}$. The sequences $\beta(r)$ representing their citations are

$$
\begin{gathered}
\beta\left(r_{1}\right)=\left(N\left(r_{1}, r_{1}\right) /\left|r_{1}\right|, N\left(r_{2}, r_{1}\right) /\left|r_{1}\right|, N\left(r_{3}, r_{1}\right) /\left|r_{1}\right|\right)=(2 / 10,1 / 10,0), \\
\beta\left(r_{2}\right)=(0,8 / 20,0),
\end{gathered}
$$

and

$$
\beta\left(r_{3}\right)=(0,5 / 30,20 / 30)
$$

Let us consider now $r_{1}$ and $r_{2}$, that satisfy that $I\left(r_{1}\right) \leq I\left(r_{2}\right)$. In order to see if the relation $\beta\left(r_{1}\right) \triangleleft \beta\left(r_{2}\right)$ is satisfied, we have to compare the sequences $(2 / 10,1 / 10,0)$ and $(0,8 / 20,0)$. Writing the second one as the sum $\gamma^{1}+\gamma^{2}+$ $\gamma^{3}=(0,4 / 20,0)+(0,4 / 20,0)+(0,0,0)$, we get that

$$
\begin{gathered}
2 / 10=\beta\left(r_{1}\right)_{1} \leq \sum_{k \geq 1} \gamma_{k}^{1}=0+4 / 20+0=2 / 10, \\
1 / 10=\beta\left(r_{1}\right)_{2} \leq \sum_{k \geq 2} \gamma_{k}^{2}=4 / 20+0=2 / 10
\end{gathered}
$$

and

$$
0=\beta\left(r_{1}\right)_{3} \leq \sum_{k \geq 3} \gamma_{k}^{3}=\gamma_{3}^{3}=0
$$

Therefore, $\beta\left(r_{1}\right) \triangleleft \beta\left(r_{2}\right)$.

The same kind of calculations show that $\beta\left(r_{2}\right) \triangleleft \beta\left(r_{3}\right)$ (and so $\beta\left(r_{1}\right) \triangleleft \beta\left(r_{3}\right)$ too). This, together with $I\left(r_{1}\right) \leq I\left(r_{2}\right) \leq I\left(r_{3}\right)$, proves in particular that the list $\mathcal{R}$ has the increasing property with respect to the index $I$.

Next result shows that, under the hypothesis that the increasing property holds, we get that the associated weighted impact factor is always increasing with $I S$. We will consider the notion defined in formula (7) for any weight function $\alpha$. If $\alpha$ is a weight function in $\mathcal{R}$, we write $I S_{\alpha}$ for the weighted impact factor defined by $\alpha$, that is, if $r_{0} \in \mathcal{R}$,

$$
I S_{\alpha}\left(r_{0}\right)=\frac{1}{\left|r_{0}\right|}\left(\sum_{a \in r_{0}}\left(\sum_{r \in \mathcal{R}}\left(\sum_{c \in r} \alpha(r) e_{a}(c)\right)\right)\right) .
$$

In case $\alpha$ is defined by the values of an impact factor $I$, we also say that $I S_{\alpha}$ is defined by $I$.

Proposition 1 Let $\mathcal{R}$ be a set of journals that has the increasing property with respect to an impact factor $I S$. Then for each $r, r^{\prime} \in \mathcal{R}, I S(r)<I S\left(r^{\prime}\right)$ implies $I S_{\alpha}(r) \leq I S_{\alpha}\left(r^{\prime}\right)$, where $I S_{\alpha}$ is the weighted impact factor defined by $I S$. 
Proof Let $r, r^{\prime} \in \mathcal{R}$, and suppose that $I S(r) \leq I S\left(r^{\prime}\right)$. Then by the increasing property we have that $\beta(r) \triangleleft \beta\left(r^{\prime}\right)$. This means that $\left(\beta\left(r^{\prime}\right)_{i}\right)_{i=1}^{n}$ can be written as a sum of $n$ non-negative sequences $\sum_{j=1}^{n}\left(\gamma_{i}^{j}\right)_{i=1}^{n}=\left(\gamma_{i}\right)_{i=1}^{n}$ such that

$$
\beta(r)_{i} \leq \sum_{k \geq i} \gamma_{k}^{i}
$$

for every $i \in\{1, \ldots, n\}$. Then for a fixed $i$, taking into account that $\alpha$ is defined by $I S$, we get that $\alpha\left(r_{i}\right) \leq \alpha\left(r_{k}\right)$ for $i \leq k$, we have that

$$
\alpha\left(r_{i}\right) \beta(r)_{i} \leq \alpha\left(r_{i}\right) \sum_{k \geq i} \gamma_{k}^{i} \leq \sum_{k \geq i} \alpha\left(r_{k}\right) \gamma_{k}^{i} .
$$

Thus,

$$
\begin{aligned}
I S_{\alpha}(r) & =\sum_{i=1}^{n} \alpha\left(r_{i}\right) \beta(r)_{i} \leq \sum_{i=1}^{n} \sum_{k \geq i} \alpha\left(r_{k}\right) \gamma_{k}^{i} \leq \sum_{i=1}^{n} \sum_{k \geq 1} \alpha\left(r_{k}\right) \gamma_{k}^{i} \\
& =\sum_{k \geq 1} \alpha\left(r_{k}\right) \sum_{i=1}^{n} \gamma_{k}^{i}=\sum_{k \geq 1} \alpha\left(r_{k}\right) \beta\left(r^{\prime}\right)_{k}=I S_{\alpha}\left(r^{\prime}\right) .
\end{aligned}
$$

This proves the result.

Corollary 1 Let $\mathcal{R}$ be a list of journals with the increasing property with respect to IS. Suppose that IS increases with IF. Then $I S_{\alpha}$ also increases with $I F$, where $I S_{\alpha}$ is the weighted impact factor defined by IS.

\section{Examples: the linear dependence hypothesis in the Thomson-Reuters impact factor lists of MATHEMATICS and MEDICINE, GENERAL AND INTERNAL}

In this section we are interested in checking in four particular cases - two different impact factors and two lists of journals ordered by the standard $I F_{2}-$ if the increasing property with respect to the new indexes is satisfied with affordable deviations. Recall that this property represents the fact that, given a measure of prestige as $I S$ for the journals of a given list $\mathcal{R}$, journals with high value of $I S$ are more often cited by journals with high value of $I S$, and vice versa. We will check a specific type of dependence - linear dependence among the $I S$ of a journal and the average values of the $I F_{2}$ of the journals that it cites-, that is also related to this increasing tendency and is more concrete and easier to check than the ordering $\triangleleft$ defined in Section 4 .

Let us define formally this new property.

Definition 2 Consider a set $\mathcal{R}$ of journals and a weight function $\alpha$. Let $r_{0} \in$ $\mathcal{R}$. We define $M\left(r_{0}\right)$ as the average of the values of $\alpha$ of the journals $r$ that are cited by $r_{0} \in \mathcal{R}$ weighted by the number of papers cited in each $r$. 
The question that we analyze in this section is the following: Is there a linear dependence (with positive slope) between $M(\cdot)$ and $\alpha(\cdot)$ ? That is, is there a relation as $M(r)=p \cdot \alpha(r)+b$, —with some affordable quadratic error-, with $p>0$ ? In case this happens, we will say that the list $\mathcal{R}$ satisfies the linear dependence hypothesis with respect to $\alpha$.

We will consider two sets of journals of two different scientific areas MATHEMATICS and MEDICINE - appearing in the Thomson-Reuters Journal Citation Reports list of these areas (JCR list in what follows). As primary information, we will consider the weight $\alpha_{2}$ of the corresponding lists, that is defined as the normalized $I F_{2}$ of a given year (see Section 3.2). We will define two indexes $-I W_{p}$ and $I W_{q}-$, that involve weights in the computation of two new types of impact factors related to the prestige of the citing journal, and we are going to compute statistical estimates for them. In order to develop our analysis, we used the following methodology.

1) First we chose a list of 10 journals equi-distributed along each ThomsonReuters 2013 list. They define the list of journals $\mathcal{R}$ in each case. In the first one (MATHEMATICS) we took a journal of each 30 - starting with the third to avoid the first ones, that cannot be considered "standard" journals - , in a way that the final subset goes through all the list, that contains 302 journals. So, we took the journals (we use the abbreviations from the JCR list), ACTA MATH, J MATH ANAL APPL, J COMB THEORY A, COMMUN PUR APPL ANAL, J MATH SOC JPN, J PURE APPL ALGEBRA, OPERATORS AND MATRICES, ARS MATH CONTEMP, OSAKA J MATH, CZECH MATH J, J MATH LOG.

In the list of MEDICINE, GENERAL and INTERNAL, we took 8 journals along the list of 156 - one of each 15 - , removing also the first journals of the list. We considered the journals AM J PREV MED, QJM-INT J MED, ARCH MED SCI, TOHOKU J EXP MED, ISR MED ASSOC J, INT J OSTEOPATH MED, MED LITH, INTERNIST.

2) Choosing randomly 50 citations of articles in journals of the 2011 and 2012 impact factor lists by articles in one of the given journals selected above, we compute estimates of the following two indexes $I W_{p}$ and $I W_{q}$ for both lists (MATHEMATICS and MEDICINE). In the case of small journals, it happened that there are not 50 citations to papers in journals of the JCR list of the last two years. In this case, we have used all the citations. Both of them are related to the prestige of the citations for a given journal that can be directly translated in terms of weights as follows.

a) $I W_{p}(r)$ : the mean of the $I F_{2}$ of the journals — weighted by the number of individual articles in each of them-, that are cited by a given journal $r$ in the last two years.

b) $I W_{q}(r)$ : the mean of the counts of the citations of the journal $r$ to papers in the $I F_{2}$ list, computed as follows: if the cited article belongs to a journal that is in the first quartile, it has a weight 4 ; if it is in the second, the weight is 3 ; if in the third, weight 2 and in the fourth, 
weight 1 . That is, if $r$ is a given journal, its weight is computed as

$$
I W_{q}(r)=\frac{1}{N(r)}\left(\sum_{r_{i} \in Q_{1}} 4+\sum_{r_{i} \in Q_{2}} 3+\sum_{r_{i} \in Q_{3}} 2+\sum_{r_{i} \in Q_{4}} 1\right),
$$

where each term represents the number of citations in the set $\mathcal{N}(r)$ of total cites in $r$ to papers published in journals that are in the quartile $Q_{j}$ of the JCR list, $j=1, \ldots, 4$, and $N(r)=|\mathcal{N}(r)|$ is the total number of citations.

Note that we have not studied what happens when this relation does not work exactly but with a small error - that is, we are not considering statistical deviation - . In other words, we assume that this error is not going to affect the general behavior of the new weighted indexes in a deep way.

The results are explained in what follows.

\subsection{MATHEMATICS}

Our first comment regarding the case of the journals of pure mathematics is the already known fact that the 2-year impact factor gives a poor measure of the prestige of a journal for the scientific community (see Arnold and Fowler (2011); Raghunathan and Srinivas (2001)). We have found again that the number of citations by papers published in 2013 to papers in the JCR lists of 2011 and 2012 is so small that it might not be statistically significant. For example, 35 papers published in the journal OPERATORS AND MATRICES in 2013 cited 819 papers, and only 50 of them were papers published in 2011 and 2012 in journals appearing in the corresponding impact factor lists.

However, and taking into account this fact, we can also notice that the linear dependence hypothesis is not very well-satisfied. Figure 1 shows that, although a linear increasing dependence can be noted between the $I F_{2}$ of the citing journal and the weighted mean $I W_{p}$ of the $I F_{2}$ of the cited journals, some particular publications do not follow this behavior. In our opinion, this is due to the fact that there are essentially two extreme types of journals appearing in the list.

1) The first one is defined by specialized publications that are devoted to some particular subjects. For example, a mathematician working in a certain specific field $A$ knows that there are for example 4 natural journals to publish her/his work. This implies that she/he tends to publish in these journals and cite papers in these journals, and so the group of these specialized journals form a closed network. Therefore, the values of their $I F_{2}$ and their position in the list depend on the size of the scientific community interested in the topic, that can be small. This produces the effect that cited journals in one of these groups have similar impact than the citing ones, following our hypothesis.

2) However, there is a second group of journals of general scope, publishing papers in different topics, some of them being popular and other ones not being 
so. The values of the impact factors of the citations of such journals will not depend on the position of the journal itself in the same list. This behavior goes against our hypothesis on the linearity of the dependence between the position in the list of the journal and the mean position of its citations.

Also, we must take into account that, by definition, all journals tend to cite papers of the top part of the impact factor list. Therefore, summing up the statistical influence of both classes of journals, one can expect an increasing behavior - high $I F_{2}$ journals cite more often high $I F_{2}$ journals-, exactly as we have, but with a high dispersion.

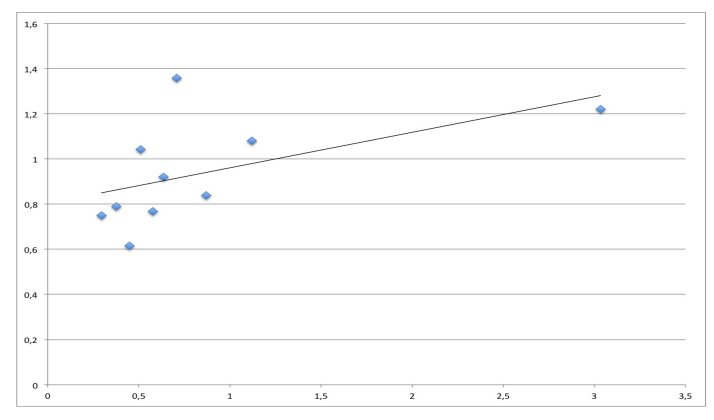

Fig. $1 I W_{p}$ of a given journal $r$ of mathematics vs the $I F_{2}$ of $r$.

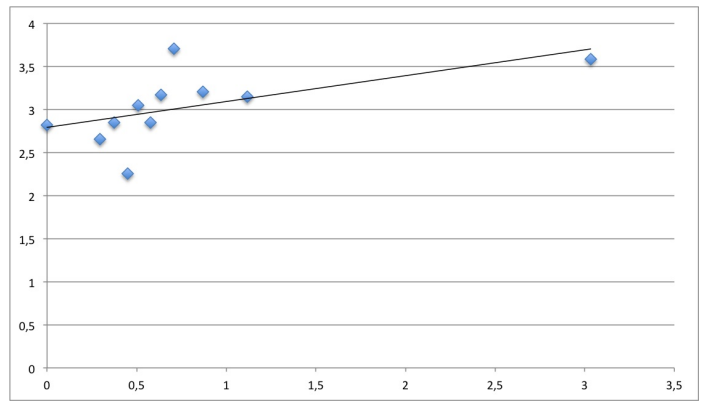

Fig. $2 I W_{q}$ of a given journal $r$ of mathematics vs the $I F_{2}$ of $r$.

Note that the second prestige-based index that we have used $\left(I W_{q}\right)$ almost satisfies the linearity hypothesis if two journals are eliminated in the graphic; we must notice that both of them belong to the second group explained in the point 2) above. 


\subsection{MEDICINE}

Usually, the experts in information science agree that $I F_{2}$ gives a reasonable measure of the prestige of a publication in the field MEDICINE (see Saha et al. (2003); Aleixandre Benavent et al. (2007)). Regarding the correlation among the $I F_{2}$ of the citing journals and the $I F_{2}$ of the cited ones, the linear fit of the points for $I W_{p}$ is clearly not satisfied for two particular journals. However, $I W_{q}$ behaves almost linearly. (See the graphics below. In both figures the highest point is not represented, since it appears far away of the rest of the points and distorts the pictures).

It can be observed that a relevant amount of citations in scientific papers lead to papers published in the last two years in journals appearing in $I F_{2}$ lists; a usual ratio is $1 / 5$, whereas in mathematics the ratios are often smaller than $1 / 10$. However, there are journals that do not follow a clear pattern.

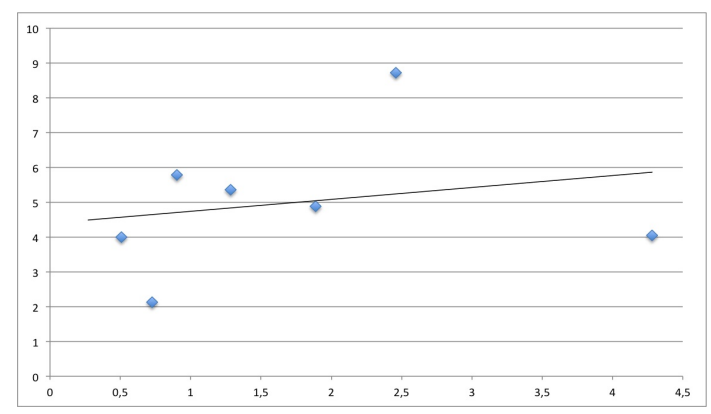

Fig. $3 I W_{p}$ of a given journal $r$ of medicine vs the $I F_{2}$ of $r$.

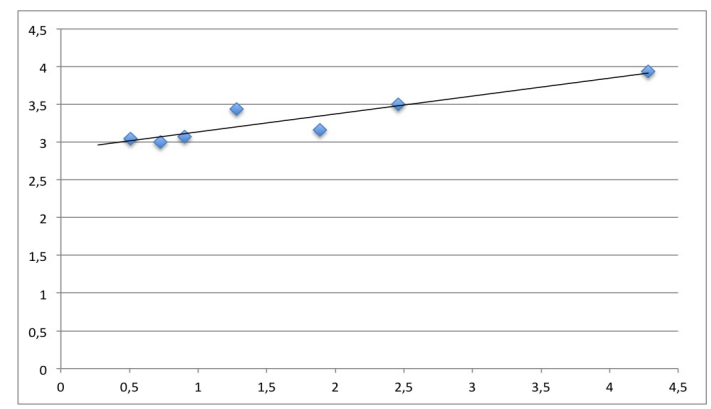

Fig. $4 I W_{q}$ of a given journal $r$ of medicine vs the $I F_{2}$ of $r$.

The linear increasing behavior can also be observed. As in the case of mathematics, the linearity hypothesis is better satisfied for $I W_{q}$ than for $I W_{p}$. 


\subsection{Discussion}

Let us finish this section by providing a brief discussion of the results obtained, and about how these results may affect to the ordering of the lists of journals with respect to the new weighted impact factors. We have shown two examples of weighted impact factors $-I W_{p}$ and $I W_{q}-$, with weights defined using two different criteria, both of them related to the prestige of the citing journals based on the index $I F_{2}$. As we said, in both cases the linear dependence hypothesis is (weakly) satisfied, although the second one gives better results. This can be translated in the following practical tool: in the first case, the new ordering of journals produced by the new weighted impact factor $I W_{p}$ may be meaningfully different than the one given by the original $I F_{2}$. However, the analyst may expect that this does not happen regarding the second weighted impact factor $I W_{q}$. In this case, the ordering of the list based on this weighted impact factor will be similar to the original one.

\section{An analysis of the error committed when the ordering based in $I F_{2}$ is changed by the ordering defined by $I S_{2}$}

We use the estimate provided by formula (8) of Section 3 for showing the absolute bound for the error committed. The bound obtained in (6) should also be used for a finer analysis. Note that the same relations hold if $I F_{2}$ is replaced by an impact factor $I S$ for a set $\mathcal{R}$ that produces a weight $\alpha$, and $I S_{2}$ is replaced by $I S_{\alpha}$ for this $\alpha$.

The triangular inequality and this estimate provides the following equivalence inequalities.

$$
\begin{gathered}
I S_{2}\left(r_{0}\right) \leq I F_{2}\left(r_{0}\right)+\left|I F_{2}\left(r_{0}\right)-I S_{2}\left(r_{0}\right)\right| \\
\leq I F_{2}\left(r_{0}\right)+I F_{2}\left(r_{0}\right) \cdot \max _{r \in \mathcal{R}}\left(\left|\alpha_{2}(r)-1\right|\right) \\
\leq I S_{2}\left(r_{0}\right)+\left|I F_{2}\left(r_{0}\right)-I S_{2}\left(r_{0}\right)\right|+\max _{r \in \mathcal{R}}\left|\alpha_{2}(r)-1\right| \cdot I F_{2}\left(r_{0}\right) \\
\leq I S_{2}\left(r_{0}\right)+2 \max _{r \in \mathcal{R}}\left|\alpha_{2}(r)-1\right| \cdot I F_{2}\left(r_{0}\right) .
\end{gathered}
$$

Therefore, when the term $\max _{r \in \mathcal{R}}\left|\alpha_{2}(r)-1\right| \cdot I F_{2}\left(r_{0}\right)$ is small enough, we obtain that the values of $I S_{2}\left(r_{0}\right)$ and $I F_{2}\left(r_{0}\right)$ are similar. The possible factors that would reduce the value of the terms are the following.

1. The value of $I F_{2}\left(r_{0}\right)$ is small. This does not affect to the relative value of the difference, but it may reduce the error.

2. The maximum of the difference of the normalized impact factor $\alpha_{2}$ and 1 is small. That is, all the journals considered in the citing set $\mathcal{R}$ have similar impact factor. This maybe obtained by using just a selected subset of citing journals instead of the whole $I F_{2}$ list. 
However, we are interested in how this error term - that can also be estimated directly for particular $I F_{2}$ lists - , produces a change in the ordering of the journals. This may happen when for a particular pair of journal $r_{1}$ and $r_{2}$ with $I F_{2}\left(r_{1}\right)<I F_{2}\left(r_{2}\right)$, we have that $I S_{2}\left(r_{1}\right) \geq I S_{2}\left(r_{2}\right)$.

Note that under the hypothesis on $I F$ for this pair of journals, formula (8) gives

$$
\begin{aligned}
I S_{2}\left(r_{1}\right) \leq & I F_{2}\left(r_{1}\right)+\left|I S_{2}\left(r_{1}\right)-I F_{2}\left(r_{1}\right)\right|<I F_{2}\left(r_{2}\right)+\left|I S_{2}\left(r_{1}\right)-I F\left(r_{1}\right)\right| \\
& \leq I S_{2}\left(r_{2}\right)+\left|I S_{2}\left(r_{2}\right)-I F\left(r_{2}\right)\right|+\left|I S_{2}\left(r_{1}\right)-I F_{2}\left(r_{1}\right)\right| \\
\leq & I S_{2}\left(r_{2}\right)+2 \max \left\{\left|I S_{2}\left(r_{2}\right)-I F\left(r_{2}\right)\right|,\left|I S_{2}\left(r_{2}\right)-I F_{2}\left(r_{2}\right)\right|\right\} \\
& \leq I S_{2}\left(r_{2}\right)+2 \max _{r \in \mathcal{R}}\left|\alpha_{2}(r)-1\right| \cdot \max \left\{I F_{2}\left(r_{1}\right), I F_{2}\left(r_{2}\right)\right\} .
\end{aligned}
$$

Therefore, we obtain the following general

Observation. If for every pair of journals $r_{1}$ and $r_{2}$ with $I F_{2}\left(r_{1}\right)<I F_{2}\left(r_{2}\right)$, we have that

$$
2 \max _{r \in \mathcal{R}}\left|\alpha_{2}(r)-1\right| \cdot \max \left\{I F_{2}\left(r_{1}\right), I F_{2}\left(r_{2}\right)\right\} \leq\left|I S_{2}\left(r_{1}\right)-I S_{2}\left(r_{2}\right)\right|
$$

then $I S_{2}\left(r_{1}\right)<I S_{2}\left(r_{2}\right)$.

To see this, assume that this does not happen. Then using (10) we obtain

$$
\begin{gathered}
I S_{2}\left(r_{1}\right)-I S_{2}\left(r_{2}\right)=\left|I S_{2}\left(r_{1}\right)-I S_{2}\left(r_{2}\right)\right| \\
\geq 2 \max _{r \in \mathcal{R}}\left|\alpha_{2}(r)-1\right| \cdot \max \left\{I F_{2}\left(r_{1}\right), I F_{2}\left(r_{2}\right)\right\}>I S_{2}\left(r_{1}\right)-I S_{2}\left(r_{2}\right),
\end{gathered}
$$

a contradiction.

Summing up these formal results, we have that the statistical changes produced by the perturbations of the linear dependence of the weights can be controlled using a max-type estimate or a square-type estimate. After getting a good estimate of a new weighted impact factor, it automatically provides a bound for the estimate of the difference between two particular journals. It can be used to obtain an interval satisfying that, if the values of $I F_{2}$ of two journals differ in more than the size of this interval, then the ordering between these two journals given by the $I F_{2}$ list is the same as the order given by the new weighted impact factor. 


\section{Conclusions}

We have introduced a general model for measuring the impact of scientific datasets, papers and journals, that we identify with an abstract version of the already used weighted factors for the measuring of the impact based on the average number of citations. In this case, a weight depending on a measure of prestige of the citing journal is given to each citation in the final computation of the impact factor. We have shown that our abstract model can be adapted to provide a formal support for the definition of citation indexes for data sets, giving for example new tools for measuring the impact of repositories of open data. We have also shown some inequalities that can provide security intervals for estimating if a new impact factor provides a different ordering in a list than the one given by the associated standard $I F$.

We have centered our attention in the analysis of what we call the increasing property for an impact factor list. A list $\mathcal{R}$ associated to an impact factor $I S$ has the increasing property if journals with high $I S$ are more often cited by journals with high $I S$, and reciprocally, journals with low $I S$ are more often cited by journals with low $I S$. We have defined an order relation $\triangleleft$ for comparing journals in order to give a precise formulation of this concept and we have proved the main result of the paper, that is given in Proposition 1: in an impact factor list has the increasing property for an index $I S$, then $I S_{\alpha}$ increases with $I S$, where the weight $\alpha$ is defined by the same $I S$. In other words, the ordering induced in the list by the values of $I S$ coincides with the one induced by the weighted impact factor $I S_{\alpha}$ associated to $\alpha$.

In the second part of the paper, we have explored the increasing property in four particular cases, for two indexes defined by means of the 2-year ThomsonReuters $I F_{2}$. We have shown that a certain linear relation between the $I F_{2}$ of the citing journals and the $I F_{2}$ of the cited journals can be observed in two impact factor lists of two scientific fields of absolutely different nature (MATHEMATICS and MEDICINE). This means that, although with a big dispersion, the increasing property for the citation lists is satisfied for these particular indexes, but the results are better for the second index $I W_{q}$ and for the list of MEDICINE. Our theoretical results imply that the final ordering of the corresponding lists provided by these new indexes - in which $I F_{2}$ is used for defining the new weight-, will be similar to the order given by $I F_{2}$.

\section{References}

1. Altmann, KG, Gorman, GE. (1998). The usefulness of impact factor in serial selection: $A$ rank and mean analysis using ecology journals. Library Acquisitions-Practise and Theory, 22, 147-159.

2. Garfield, E. (2006). The History and Meaning of the Journal Impact Factor. JAMA. 295(1), 90-93. 
3. Pinto, A.C., Andrade, J.B. (1999). Impact factor of scientific journals: What is the meaning of this parameter? Quimica Nova, 22, 448-453.

4. Saha, S, Saint, S, Christakis, D.A. (2003). Impact factor: a valid measure of journal quality? Journal of the Medical Library Association, 91, 42-46.

5. Buela-Casal, G. (2003). Evaluating quality of articles and scientific journals. Proposal of weighted impact factor and a quality index. Psicothema, 15(1), 23-25.

6. Habibzadeh, F., Yadollahie, M. (2008). Journal weighted impact factor: A proposal. Journal of Informetrics, 2(2), 164-172.

7. Waltman, L., van Eck, N.J. (2008). Some comments on the journal weighted impact factor proposed by Habibzadeh and Yadollahie. Journal of Informetrics, 2(4), 369-372.

8. Zitt, M, Small, H. (2008). Modifying the journal impact factor by fractional citation weighting: The audience factor. Journal of the American Society for Information Science and Technology, 59, 1856-1860.

9. Pinski, G, Narin, F. (1976). Citation influence for journal aggregates of scientific publications: theory, with application to the literature of physics. Information Processing and Management, 12, 297-312.

10. Zyczkowski, K. (2010). Citation graph, weighted impact factors and performance indices. Scientometrics, 85(1), 301-315.

11. Leydesdorff, L., Opthof, T. (2010). Scopus's Source Normalized Impact per Paper (SNIP) Versus a Journal Impact Factor Based on Fractional Counting of Citations. Journal of the American Society for Information Science and Technology, 61, 2365-2369.

12. Moed, H.F. (2010). Measuring contextual citation impact of scientific journals. Journal of Informetrics, 4, 265-277.

13. NISO. Alternative Metrics Initiative Phase 1. White Paper. (2014). http://www.niso.org/apps/group-public/download.php/13809/Altmetrics-projectphase1-white-paper.pdf

14. Torres-Salinas, D., Jimenez-Contreras, E. (2010). Introduction and comparative study of the new scientific journals citation indicators in Journal Citation Reports and Scopus. El Profesional de la Información, 19, 201-207.

15. Waltman, L., van Eck, N.J, van Leeuwen, TN., Visser, MS. (2013). Some modifications to the SNIP journal impact indicator. Journal of Informetrics, 7, 272-285.

16. Zitt, M. (2011). Behind citing-side normalization of citations: some properties of the journal impact factor. Scientometrics, 89, 329-344.

17. Ahlgren, P., Waltman, L. (2014). The correlation between citation-based and expertbased assessments of publication channels: SNIP and SJR vs. Norwegian quality assessments. Journal of Informetrics, 8, 985- 996.

18. Aleixandre Benavent, R., Valderrama Zurián, J.C., González Alcaide, G. (2007). Scientific journals impact factor: limitations and alternative indicators. El Profesional de la Información, 16(1), 4-11.

19. Dorta-Gonzalez, P., Dorta-Gonzalez, M.I. (2013). Comparing journals from different fields of science and social science through a JCR subject categories normalized impact factor. Scientometrics, 95(2), 645-672.

20. Dorta-Gonzalez, P., Dorta-Gonzalez, M.I., Santos-Penate, D.R., Suarez-Vega, R. (2014). Journal topic citation potential and between-field comparisons: The topic normalized impact factor, Journal of Informetrics, 8(2), 406-418.

21. Egghe, L., Rousseau, R. (2002). A general frame-work for relative impact indicators. Canadian Journal of Information and Library Science, 27(1), 29-48.

22. Li, Y.R., Radicchi, F., Castellano, C., Ruiz-Castillo, J. (2013). Quantitative evaluation of alternative field normalization procedures. Journal of Informetrics, 7(3), 746-755.

23. Owlia, P., Vasei, M., Goliaei, B., Nassiri, I. (2011). Normalized impact factor (NIF): An adjusted method for calculating the citation rate of biomedical journals. Journal of Biomedical Informatics, 44(2), 216-220.

24. Ruiz Castillo, J., Waltman, L. (2015). Field-normalized citation impact indicators using algorithmically constructed classification systems of science. Journal of Informetrics, 9 102-117.

25. Arnold, D. N., Fowler, K.K. (2011). Nefarious numbers. Notices of the American Mathematical Society, 58(3), 434-437. 
26. Raghunathan, M.S., Srinivas, V. (2001). Significance of impact factor with regard to mathematics journals. Current Science, 80(5), 605. 